\title{
Correlação dos aspectos clínicos e questionários do sono com achados polissonográficos em pacientes com apnéia obstrutiva do sono
}

\author{
Correlation of clinical aspects and sleep questionnaires with polysonographic findings in \\ patients with obstructive sleep apnea \\ Correlación de aspectos clínicos y cuestionarios del sueño com hallazgos \\ polisomnográficos en pacientes con apnea obstructiva del sueño
}

Jocyane de Souza Andrade ${ }^{1 *}$, José Roberto Bentes Capeloni ${ }^{1}$, Mateus Pantoja Rocha ${ }^{2}$, Felipe Xavier de Souza ${ }^{1}$, Ana Larisse Gondim Barbosa1, André dos Santos Brandão1.

\begin{abstract}
RESUMO
Objetivo: Correlacionar alterações clínicas e questionários de sono com achados polissonográficos em pacientes com Apnéia Obstrutiva do Sono (AOS) atendidos em ambulatório de sono de um hospital de referência. Métodos: Estudo observacional, transversal, realizado em um hospital de referência em otorrinolaringologia. Foram selecionados 35 pacientes com queixa de roncos e que já haviam realizado polissonografia. Os pacientes preencheram três questionários de sono: Escala de Sonolência de Epworth (ESE), Questionário de Berlim e Questionário STOP-BANG. Resultados: AOS foi encontrada em $83 \%$ dos pacientes, sendo $55,2 \%$ homens e $44,8 \%$ mulheres, maioria na sexta década de vida (41.4\%). A média do Índice de Massa Corporal (IMC) foi de 32,6 ( \pm 4,9 DP) e da circunferência cervical, foi de $39,7 \mathrm{~cm}( \pm 4,9$ DP). Quanto à sensibilidade e especificidade dos questionários em predizer risco de AOS, obteve-se que a ESE apresentou sensibilidade de $68,97 \%$ e especificidade de $33,33 \%$, STOP-BANG apresentou sensibilidade $93.1 \%$ e especificidade $16,6 \%$ e o Questionário de Berlim, sensibilidade 96,5\% e especificidade $0 \%$. Conclusão: Os questionários ESE, Berlim e STOP-BANG, identificaram pacientes de alto risco para AOS, mas não excluíram com precisão os de baixo risco.
\end{abstract}

Palavras-chave: Apnéia obstrutiva do sono, Inquéritos e questionários, Polissonografia.

\begin{abstract}
Objective: To correlate clinical changes and sleep questionnaires with polysomnographic findings in patients with obstructive sleep apnea (OSA) in a medical care sleep clinic of a reference hospital. Methods: This was an observational, cross-sectional study conducted at a referral hospital for otolaryngology. Thirty-five patients who complained of snoring and who had already performed polysomnography were selected. The patients completed three sleep questionnaires: Epworth Sleepiness Scale (ESE), Berlin Questionnaire and STOPBang Questionnaire. Results: OSA was found in $83 \%$ of patients, $55.2 \%$ men and $44.8 \%$ women, most in the sixth decade of life (41.4\%). The mean Body Mass Index (BMI) was 32.6 ( \pm 4.9 PD) and cervical circumference was $39.7 \mathrm{~cm}( \pm 4.9 \mathrm{PD})$. Regarding the sensitivity and specificity of the questionnaires in predicting OSA risk, it was found that the ESE presented sensitivity of $68.97 \%$ and specificity of $33.33 \%$, STOP-BANG presented sensitivity $93.1 \%$ and specificity $16.6 \%$ and the Berlin Questionnaire, sensitivity 96.5\% and specificity 0\%. Conclusion: The ESE, Berlin and STOP-BANG questionnaires identified high risk patients for OSA, but did not accurately exclude low risk patients.
\end{abstract}

Keywords: Sleep apnea obstructive, Surveys and questionnaires, Polysomnography.

${ }_{1}$ Universidade Federal do Pará (UFPA), Belém-PA *E-mail: jocyane.andrade@gmail.com

2 Universidade do Estado do Pará (UEPA), Belém-Pará.

SUBMETIDO EM: 1/2020 


\section{RESUMEN}

Objetivo: Correlacionar alteraciones clínicas y cuestionarios del sueño com hallazgos polisomnográficos en pacientes com apnea obstructiva del sueño (AOS) atendidos en ambulatório del sueño de hospital de referencia. Métodos: Estudio observacional, transversal realizado en hospital de otorrinolaringología. Fueron seleccionados 35 pacientes que se quejaban de ronquidos y que ya habían realizado polisomnografía. Fueron rellenados três cuestionarios de sueño: Escala de somnolencia de Epworth (ESE), cuestionario de Berlín y cuestionario STOP-Bang. Resultados: AOS se encontro en $83 \%$ pacientes, $55.2 \%$ hombres y $44.8 \%$ mujeres, la mayoría en la sexta década de vida (41.4\%). El índice de masa corporal (IMC) medio fue 32,6 $( \pm 4,9 \mathrm{DP})$ y la circunferencia cervical fue $39,7 \mathrm{~cm}( \pm 4,9 \mathrm{DP})$. Con respecto a sensibilidad y especificidad de los cuestionarios para predecir riesgo de AOS, se encontró que ESE presentaba una sensibilidade del $68,97 \%$ y una especificidade del $33,33 \%$, STOP-BANG presentaba una sensibilidade del $93,1 \%$ y una especificidad del $16,6 \%$ y cuestionario Berlín, sensibilidad $96.5 \%$ y especificidad $0 \%$. Conclusión: Los cuestionarios ESE, Berlín y STOP-BANG identificaron pacientes de alto riesgo para OSA, pero no excluyeron con precisión los de bajo riesgo.

Palabras clave: Apnea obstructiva del sueño, Encuestas y cuestionarios, Polisomnografía.

\section{INTRODUÇÃO}

A apnéia obstrutiva do sono (AOS) é caracterizada por episódios repetidos de obstrução das vias aéreas superiores durante o sono, ocasionando hipoxemia, hipercapnia e fragmentação do sono (PARK JG, et al., 2011). A AOS é um transtorno respiratório do sono bastante comum, afetando cerca de $2-4 \%$ da população adulta (MANNARINO MR, et al., 2012). Um estudo epidemiológico realizado na cidade de São Paulo, observou que a prevalência da AOS foi de 32,9\% (TUFIK S, et al., 2010).

Recentemente, estudo realizado em Lausanne (Suíça), entre 2009 e 2013, denominado HypnoLaus, observou uma prevalência de $23,4 \%$ em mulheres e de 49,7\% em homens, com gravidade entre moderada à grave (HEINZER R, et al., 2015). Estima-se que AOS não tratada tenha custos médicos adicionais de até US $\$ 3,4$ bilhões por ano nos EUA (PEPPARD PE, et al., 2013).

Os estudos populacionais são unâmimes em estimar que a prevalência da AOS é maior em diferentes subgrupos, incluindo sexo masculino, indivíduos mais velhos e aqueles com sobrepeso ou obesidade (BIXLER EO, et al., 2001; IP MS, et al., 2004; KIM J, et al, 2004). Estima-se que a maioria dos pacientes não foram diagnosticados devido à falta de sensibilidade e especificidade das manifestações clínicas, problemas de acessibilidade aos serviços de saúde e altos custos do estudo diagnóstico (PEPPARD PE, et al., 2013).

O método de diagnóstico padrão ouro é a polissonografia (PSG). PSG é uma técnica não invasiva que envolve a monitorização durante a noite de variáveis fisiológicas, incluindo eletroencefalografia, movimentos oculares, tônus muscular, bem como esforço respiratório, fluxo de ar e saturação de oxigênio (BERRY RB, et al., 2018). Considerando o alto custo e a inacessibilidade do PSG, vários questionários de triagem foram desenvolvidos. Dentre os questionários mais populares tem-se: o Questionário de Berlim, Questionário STOP-BANG e Escala de Sonolência de Epworth (ESE).

Nos últimos anos, a precisão dos questionários sobre AOS tem sido uma área de crescente interesse de pesquisa e vários estudos foram publicados sobre o assunto (AMRA B, et al.,2018; PEÑAFIEL FS, et al; PRASAD KT, et al., 2017). Com o crescente número de encaminhamentos para centros especializados, esses métodos de rastreamento são progressivamente mais importantes. Variáveis antropométricas, sintomas auto-relatados, demografia e comorbidades têm sido usados para desenvolver numerosos modelos de previsão clínica.

Diante ao impacto do atraso no diagnóstico e tratamento de pacientes com AOS, bem como baixa acessibilidade da PSG, métodos alternativos de triagem se fazem necessários, a exemplo dos questionários de sono e análise dos preditores de risco. Este estudo objetivou correlacionar as alterações clínicas e 
questionários do sono com achados polissonográficos em pacientes com AOS atendidos em ambulatório de sono de um hospital de referência.

\section{MÉTODOS}

Trata-se de um estudo observacional, com desenho transversal, realizado em pacientes atendidos no ambulatório de sono do serviço de otorrinolaringologia do Hospital Universitário Bettina Ferro de Souza (HUBFS), de Belém-Pará, no período de setembro a novembro de 2019.

Foram selecionados para o estudo 35 pacientes atendidos no ambulatório do sono com queixas de ronco e que realizaram a polissonografia. Para cada paciente atendido no ambulatório de sono foi realizado o preenchimento de protocolo específico contendo informações de dados de exame físico e mais três questionários de sono: Escala de Sonolência de Epworth, Questionário de Berlim e Questionário STOPBANG.

O Questionário de Berlim permite estimar o risco de apresentar distúrbios respiratórios do sono na população adulta. A primeira categoria inclui cinco perguntas sobre ronco e apnéias presenciadas. A segunda categoria inclui quatro questões sobre sonolência diurna. A terceira categoria questiona história de hipertensão arterial e obesidade. Um paciente é considerado em alto risco de desenvolver AOS quando ele tem duas ou mais categorias positivas (PEÑAFIEL FS, et al., 2018).

O Questionário STOP- BANG consiste em quatro perguntas que avaliam a presença de ronco intenso, sonolência diurna ou fatigabilidade, apnéias presenciadas durante o sono e hipertensão arterial, idade, sexo, índice de massa corporal e circunferência cervical. Os sujeitos com alto risco de AOS são identificados quando respondem afirmativamente pelo menos trêsquestões (NAGAPPA $M$ et al., 2015; DUARTE RLM, et al., 2017).

A Escala de Sonolência de Epworth (ESE) é questionário auto-aplicável que solicita aos pacientes que avaliem a probabilidade de adormecer em oito situações ou atividades específicas da vida diária. Ele qualifica-se numa escala de 0 a 3 pontos em cada situação e a pontuação pode variar de 0 a 24 . A ESE é classificada como: baixo risco de Sonolência Excessiva Diurna (menos de 11 pontos) e de alto risco de Sonolência Excessiva Diurna (11-24 pontos), que neste estudo foi considerado alto risco para AOS (JOHNS MW, 1991).

O exame polissonográfico noturno foi registrado no sistema Brain Wave (NeuroVirtual) ${ }^{\circledR}$, utilizando os registros de EEG: F4-M1, C4-M1, O2-M1, F3-M2, C3-M2, O1-M2; EOG: bilateral; EMG: mentoniano/submentoniano; EMG: tibial bilateral; ECG: montagem D2 modificada; fluxo aéreo nasal por cânula de pressão e oronasal por termístor; esforço respiratório torácico e abdominal; saturação da oxihemoglobina (SpO2) por oximetria de pulso; sensor de ronco (microfone) e posição corporal no leito.

Foram excluídos do estudo: pacientes que não responderam ao protocolo de pesquisa no dia da consulta; não aceitaram participar da pesquisa; pacientes que não realizaram a polissonografia ou cujo prontuário não foi localizado.

Os dados da pesquisa foram quantificados descritivamente sob a forma de gráficos e tabelas utilizando 0 software Microsoft Office Word e Microsoft Office Excel.As análises estatísticas foram realizadas no GraphPad Prisma 6.0. As estatísticas descritivas foram apresentadas por meio de frequências (absoluta e relativa), médias e desvio-padrão.

Cada questionário foi comparado com os seguintes parâmetros: sensibilidade, especificidade, valores preditivos positivos (VPP), valores preditivos negativos (VPN) e razão de verossimilhança. Também foi usado Teste Exato de Fisher, teste $G$ e teste de Kruskall- Walls para cálculo do $p$ valor. $O$ nível de significância utilizado foi de $5 \%$.

O estudo foi iniciado após aprovação do Comitê de Ética em Pesquisa, sob número de parecer 3600.836 e CAAE: 19437819.2.0000.0017, em consonância com a Resolução 466/2012 do Conselho Nacional de Saúde que regulamenta as questões operacionais e éticas dos trabalhos científicos envolvendo seres 
humanos. Os pacientes assinaram o Termo de Consentimento Livre e Esclarecido (TCLE) para acesso as informações de prontuário e autorização da pesquisa.

\section{RESULTADOS}

Foram avaliados 35 pacientes atendidos no ambulatório de sono do Hospital Bettina Ferro de Souza, que responderam aos questionários do sono e realizaram a polissonografia. As características clínicas e demográficas dos pacientes do estudo estão apresentadas na Tabela 1.

Neste estudo, $48.6 \%$ eram homens e $51.4 \%$ mulheres, entre terceira e sétima década de vida. Nos pacientes com AOS a maioria eram homens (55.1\%), sobretudo no grupo grave $(70 \%)$. Com relação a idade a maioria dos pacientes com AOS se encontrava na sexta década de vida (41.4\%). Não houve diferença significativa entre a média de idade dos pacientes dentre os grupos da AOS.

Com relação às co-morbidades, $62,9 \%$ apresentavam alguma doença, sendo a hipertensão arterial a mais frequente (48.6\%), seguido pela Diabetes Melitus (17,1\%). Arritmia, AVC, hipotireoidismo e insuficiência cardíaca estiveram presentes em 2,9\% dos pacientes. Quanto às horas de sono dormidas por noite, $45.7 \%$ tinha em torno de 6-8 horas de sono por noite.

O Gráfico 1 mostra as queixas mais frequentes como presença de ronco (100\%), sonolência diurna (91.4\%) e despertares noturnos (88.6\%).

Tabela 1 - Características clínicas e demográficas da população de estudo, segundo grupos de Apnéia Obstrutiva do Sono, n=35. Belém-PA,2019.

\begin{tabular}{|c|c|c|c|c|c|c|c|c|c|c|}
\hline & Total & & AOS & & $\begin{array}{l}\text { AOS } \\
\text { Leve }\end{array}$ & & $\begin{array}{c}\text { AOS } \\
\text { Moderada }\end{array}$ & & $\begin{array}{l}\text { Aos } \\
\text { Grave }\end{array}$ & \\
\hline & $n=35$ & $\%$ & & $\%$ & $n=6$ & $\%$ & $n=3$ & $\%$ & $\mathrm{n}=20$ & $\%$ \\
\hline \multicolumn{11}{|l|}{ Sexo } \\
\hline Fem & 18 & 51.4 & 13 & 44.8 & 5 & 83.3 & 2 & 66.7 & 6 & 30.0 \\
\hline Masc & 17 & 48.6 & 16 & 55.2 & 1 & 16.7 & 1 & 33.3 & 14 & $70.0^{*}$ \\
\hline \multicolumn{11}{|l|}{ Idade } \\
\hline 28 a 49 & 14 & 40.0 & 9 & 31.0 & 1 & 16.7 & 1 & 33.3 & 7 & $35.0^{\#}$ \\
\hline 50 a 59 & 13 & 37.1 & 12 & 41.4 & 5 & 83.3 & 2 & 66.7 & 5 & 25.0 \\
\hline$>=60$ & 8 & 22.9 & 8 & 27.6 & 0 & 0.0 & 0 & 0.0 & 8 & 40.0 \\
\hline \multicolumn{11}{|l|}{ Horas de sono } \\
\hline Até 6 horas & 14 & 40.0 & 12 & 41.4 & 2 & 33.3 & 1 & 33.3 & 9 & $45.0^{\#}$ \\
\hline 6 a 8 horas & 16 & 45.7 & 14 & 48.3 & 2 & 33.3 & 2 & 66.7 & 10 & 50.0 \\
\hline$>8$ horas & 5 & 14.3 & 3 & 10.3 & 2 & 33.3 & 0 & 0.0 & 1 & 5.0 \\
\hline \multicolumn{11}{|c|}{ Comorbidades } \\
\hline Sim & 22 & 62.9 & 17 & 58.6 & 4 & 66.7 & 2 & 66.7 & 11 & 55.0 \\
\hline Não & 13 & 37.1 & 12 & 41.4 & 2 & 33.3 & 1 & 33.3 & 9 & 45.0 \\
\hline
\end{tabular}

Legenda: ${ }^{*} p<0,05 . \quad \# p>0,05$ Fonte: Andrade JS, et al.,2019. 
Gráfico 1 - Principais queixas dos pacientes do estudo atendidos no ambulatório de sono. N=35. Belém-PA, 2019.

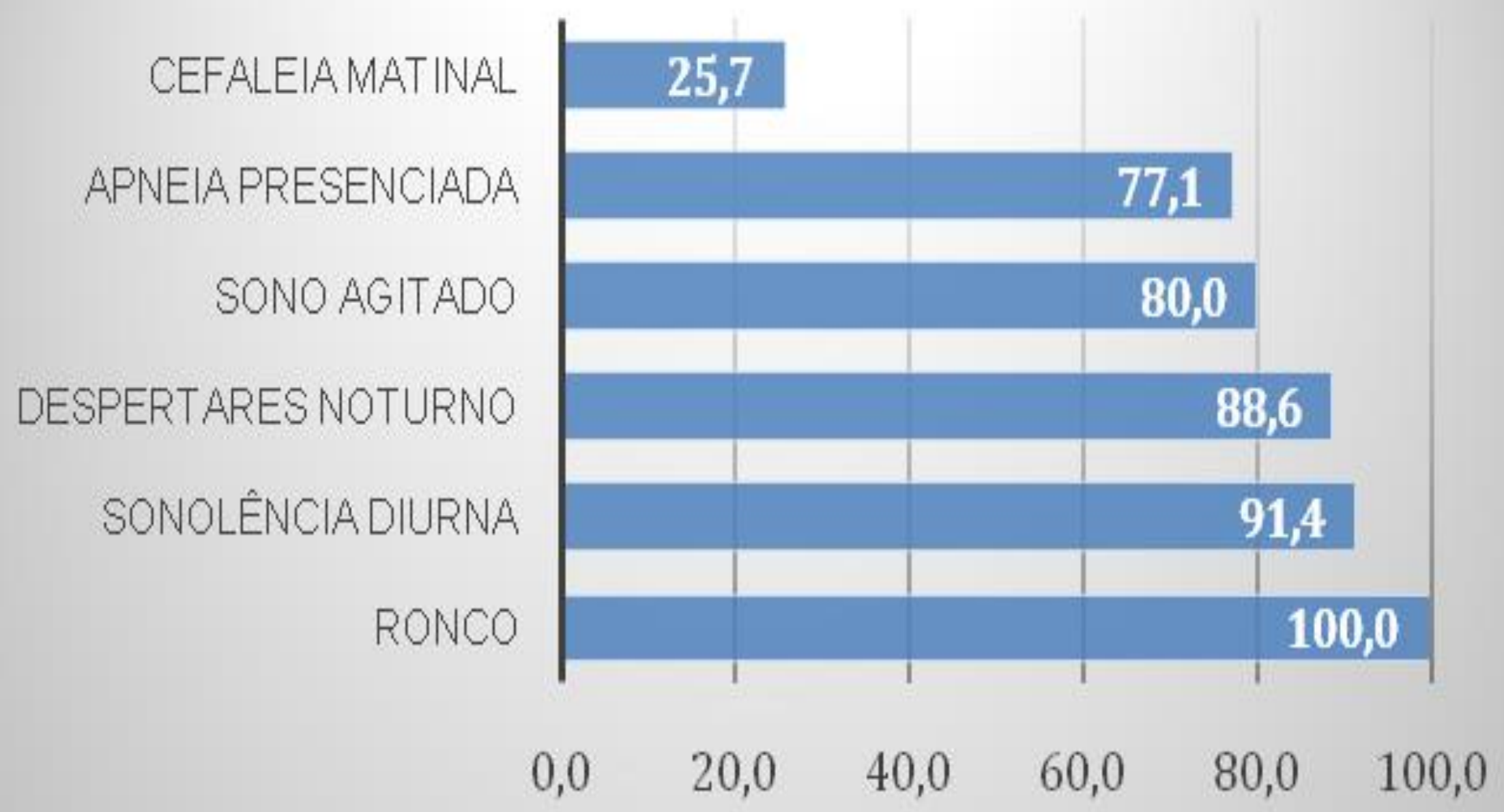

Fonte: Andrade JS, et al.,2019.

REAS/EJCH |Vol.12(3) | e2815 | DOI: https://doi.org/10.25248/reas.e2815.2020 Página $\mathbf{5}$ de $\mathbf{1 1}$ 
Através da anamnese e do estudo da polissonografia foi possível definir o diagnóstico de Apnéia Obstrutiva do Sono (AOS) em $83 \%$ dos pacientes do estudo. Foi possível definir ainda a severidade da AOS de acordo com o IAH, em que 17,1\% apresentavam AOS leve (AIH $\geq 5<15$ ), 8,5\% com AOS moderada $(\mathrm{AlH} \geq 15<30)$ e $57.1 \%$ apresentavam $\mathrm{AOS}$ grave $(\mathrm{AlH} \geq 30)$. No grupo de $\mathrm{AOS}$ grave a maioria eram homens $(70 \%)$.

A análise das características físicas dos pacientes está demonstrada na Tabela 2. Com relação à classificação de Friedman, a maioria dos pacientes apresentava classificação II e III $(25.7 \%$ e $62.9 \%$, respectivamente). A maioria dos pacientes não apresentava desvios septais obstrutivos (88.6\%) ou alterações craniofaciais significativas (94.3\%).

Com relação ao Índice de Massa Corporal (IMC), a média foi de 32,6 ( \pm 4,9 DP), sem diferença significativa entre os grupos classificados de AOS leve, moderada e grave. Com relação à circunferência cervical, a média foi de $39,7 \mathrm{~cm}( \pm 4,9 \mathrm{DP})$, com maior média no grupo AOS grave $(41,2 \mathrm{~cm})$.

Tabela 2 - Avaliação das características físicas da população estudada, segundo grupos de Apnéia Obstrutiva do Sono. N=35. Belém-PA,2019.

\begin{tabular}{|c|c|c|c|c|c|c|c|c|}
\hline & Total & & $\begin{array}{l}\text { AOS } \\
\text { Leve }\end{array}$ & & $\begin{array}{c}\text { AOS } \\
\text { Moderada }\end{array}$ & & $\begin{array}{l}\text { AOS } \\
\text { Grave }\end{array}$ & \\
\hline & $n=35$ & $\%$ & $n=6$ & $\%$ & $n=3$ & $\%$ & $n=20$ & $\%$ \\
\hline \multicolumn{9}{|l|}{ Friedman } \\
\hline II & 9 & 25.7 & 0 & 0.0 & 1 & 33.3 & 6 & 30.0 \\
\hline III & 22 & 62.9 & 5 & 83.3 & 2 & 66.7 & 11 & 55.0 \\
\hline IV & 4 & 11.4 & 1 & 16.7 & 0 & 0.0 & 3 & 15.0 \\
\hline \multicolumn{9}{|l|}{$\begin{array}{l}\text { Desvio septal } \\
\text { obstrutivo }\end{array}$} \\
\hline Sim & 4 & 11.4 & 0 & 0.0 & 1 & 33.3 & 2 & 10.0 \\
\hline Não & 31 & 88.6 & 6 & 100.0 & 2 & 66.7 & 18 & 90.0 \\
\hline $\begin{array}{c}\text { Alteração } \\
\text { crânio facial }\end{array}$ & & & & 0.0 & & 0.0 & & 0.0 \\
\hline Birretruso & 1 & 2.9 & 1 & 16.7 & 0 & 0.0 & 0 & 0.0 \\
\hline Normal & 33 & 94.3 & 5 & 83.3 & 3 & 100.0 & 19 & 95.0 \\
\hline \multirow[t]{2}{*}{ Retrognata } & 1 & 2.9 & 0 & 0.0 & 0 & 0.0 & 1 & 5.0 \\
\hline & Média & $\begin{array}{l}\text { Desvio- } \\
\text { padrão }\end{array}$ & Média & $\begin{array}{l}\text { Desvio- } \\
\text { padrão }\end{array}$ & Média & $\begin{array}{l}\text { Desvio- } \\
\text { padrão }\end{array}$ & Média & $\begin{array}{l}\text { Desvio- } \\
\text { padrão }\end{array}$ \\
\hline Cinc. Cervical (cm) & 39.7 & 4.9 & 38.4 & 6.3 & 37.5 & 0.9 & 41.2 & 4.9 \\
\hline IMC & 32.6 & 4.9 & 33.6 & 5.1 & 29.9 & 2.5 & 33.2 & 5.2 \\
\hline
\end{tabular}

Legenda: IMC: índice de massa corporal. Fonte: Andrade JS, et al.,2019. 
Na Tabela 3 estão apresentados os dados polissonográficos dos pacientes, de acordo com a gravidade da AOS. O IAH - Índice de Apnéia e hipopnéia médio foi de 35,5 eventos $/$ h $( \pm 27.7 \mathrm{DP})$. Houve atraso na Latência para sono REM (LSR) em $48,6 \%$ dos pacientes, sobretudo nos pacientes classificados com AOS grave (45\%). A Latência para início do sono (LIS) esteve reduzida em $62,9 \%$ dos pacientes, sobretudo nos pacientes classificados com AOS grave (65\%). A Eficiência do sono (TTS/TTR) também esteve reduzida (<85\%) em $57,1 \%$ dos pacientes do estudo. A média do Índice de Microdespertares (IMD) foi de $38 / \mathrm{h}$ ( $\pm 21 \mathrm{DP})$, com $16.5 / \mathrm{h}$ no grupo AOS leve, $27.3 / \mathrm{h}$ no grupo moderado e $53.1 / \mathrm{h}$ no grupo grave.

Tabela 3 - Dados polissonográficos dos pacientes, de acordo com a gravidade da AOS. $n=35$. BelémPA,2019.

\begin{tabular}{|c|c|c|c|c|c|c|c|c|}
\hline & Total & & $\begin{array}{l}\text { AOS } \\
\text { Leve }\end{array}$ & & $\begin{array}{l}\text { AOS } \\
\text { Moderada }\end{array}$ & & $\begin{array}{l}\text { AOS } \\
\text { Grave }\end{array}$ & \\
\hline & $\mathrm{n}=35$ & $\%$ & $\mathrm{n}=6$ & $\%$ & $n=3$ & $\%$ & $\mathrm{n}=\mathbf{2 0}$ & $\%$ \\
\hline \multicolumn{9}{|l|}{ IAH } \\
\hline Média & 35.5 & & 8 & & 26.7 & & 54.8 & \\
\hline Desvio-padrão & 27.7 & & 3.4 & & 13.3 & & 19.1 & \\
\hline \multicolumn{9}{|l|}{ LSR (min) } \\
\hline precoce & 6 & 17.1 & 1 & 16.7 & 1 & 33.3 & 4 & 20.0 \\
\hline normal & 12 & 34.3 & 1 & 16.7 & 2 & 66.7 & 7 & 35.0 \\
\hline tardia & 17 & 48.6 & 4 & 66.7 & 0 & 0.0 & 9 & 45.0 \\
\hline \multicolumn{9}{|l|}{ LIS (min) } \\
\hline normal & 13 & 37.1 & 4 & 66.7 & 1 & 33.3 & 7 & 35.0 \\
\hline reduzido & 22 & 62.9 & 2 & 33.3 & 2 & 66.7 & 13 & 65.0 \\
\hline \multicolumn{9}{|c|}{$\begin{array}{l}\text { Eficiência do sono } \\
\text { (TTS/TTR) }\end{array}$} \\
\hline normal & 15 & 42.9 & 2 & 33.3 & 2 & 66.7 & 7 & 35.0 \\
\hline reduzido & 20 & 57.1 & 4 & 66.7 & 1 & 33.3 & 13 & 65.0 \\
\hline \multicolumn{9}{|l|}{ IMD } \\
\hline Média & 38 & & 16.5 & & 27.3 & & 53.1 & \\
\hline Desvio-padrão & 21 & & 4.0 & & 16.5 & & 14.1 & \\
\hline \multicolumn{9}{|l|}{ IMPMI } \\
\hline Normal & 25 & 71.4 & 4 & 66.7 & 2 & 66.7 & 15 & 75.0 \\
\hline MPM leve & 1 & 2.9 & 0 & 0.0 & 0 & 0.0 & 1 & 5.0 \\
\hline MPM Moderado & 1 & 2.9 & 0 & 0.0 & 0 & 0.0 & 1 & 5.0 \\
\hline MPM Grave & 8 & 22.9 & 2 & 33.3 & 1 & 33.3 & 3 & 15.0 \\
\hline \multicolumn{9}{|l|}{ Dessaturação } \\
\hline Sim & 21 & 60.0 & 2 & 33.3 & 1 & 33.3 & 18 & 90.0 \\
\hline Não & 14 & 40.0 & 4 & 66.7 & 2 & 66.7 & 2 & 10.0 \\
\hline
\end{tabular}

Legenda: IAH: Índice de Apnéia e hipopnéia (/h). LSR: Latência para o Sono REM. LIS: Latência para Início do Sono. TTS: Tempo Total de Sono. TTR: Tempo Total de Registro. IMD: Índice de Microdespertares. IMPMI: Índice de Movimentos Periódicos de Membros Inferiores. MPM: Movimentos Periódicos dos Membros. Teste Exato de Fisher. $\mathrm{P}<0$,05. Fonte: Andrade JS, et al.,2019.

Com relação ao Índice de Movimentos Periódicos de Membros inferiores (IMPMI), 71.4\% encontravamse dentro da normalidade. A dessaturação esteve presente em $60 \%$ dos pacientes do estudo. No grupo com AOS graves $90 \%$ apresentaram dessaturação, enquanto que nos grupos AOS leve e moderado a maioria (66,7\% e 66,7\%) não apresentou dessaturações importantes. 
Na Tabela 4 é possível analisar os resultados da ESE, Questionário de Berlim e STOP-BANG de acordo com o risco para AOS. Na ESE, $68.6 \%$ pacientes obtiveram respostas com pontuação $>10$ caracterizando alto risco para AOS. A ESE conseguiu identificar $69 \%$ dos pacientes com AOS mas falhou em $31 \%$, que apresentavam AOS, mas foram classificados em baixo risco (pontuação $\leq 10$ ) pela escala. O questionário de Berlim classificou como alto risco $97,1 \%(n=34)$ dos pacientes do estudo. Dos pacientes com AOS, o questionário de Berlim identificou 96.6\% ( $n=28)$ como classificados em alto risco e 1 como baixo risco. Dentre os pacientes sem AOS, todos foram classificados previamente com alto risco pelo questionário de Berlim. De forma semelhante, o questionário STOP-BANG identificou como alto risco $93.1 \%(n=27)$ dos pacientes classificados com AOS.

Tabela 4 - Análise dos resultados de ESE, Berlim e STOP-BANG, e o risco para AOS.n=35. BelémPA,2019.

\begin{tabular}{lllllll}
\hline & Total & & Com AOS & & Sem AOS \\
\hline & $\mathbf{n = 3 5}$ & $\%$ & $\mathbf{n = 2 9}$ & $\%$ & $\mathbf{n}=\mathbf{6}$ & $\%$ \\
\hline E S E & & & & & & 66.7 \\
\hline$>10$ & 24 & 68.6 & 20 & 69.0 & 4 & 33.3 \\
$\leq 10$ & 11 & 31.4 & 9 & 31.0 & 2 & 100.0 \\
\hline Q. BERLIM & & & & & & 0.0 \\
\hline Alto risco & 34 & 97.1 & 28 & 96.6 & 6 & \\
Baixorisco & 1 & 2.9 & 1 & 3.4 & 0 & 83.3 \\
\hline Q. STOP-BANG & & & & & & 16.7 \\
\hline Alto risco & 32 & 91.4 & 27 & 93.1 & 5 & \\
Baixorisco & 3 & 8.6 & 2 & 6.9 & 1 & \\
\hline
\end{tabular}

Fonte: Andrade JS, et al.,2019.

Teste Exato de Fisher. $p<0,05$.

Na Tabela 5 está resumida a análise da sensibilidade e especificidade dos questionários ESE, Berlim e STOP-BANG em predizer o risco para AOS. A ESE apresentou sensibilidade de $68,97 \%$ e especificidade de $33,33 \%$. O questionário STOP-BANG apresentou alta sensibilidade (93.1\%) e baixa especificidade $(16,6 \%)$. O questionário de Berlim foi o que apresentou maior sensibilidade $(96,5 \%)$, porém $0 \%$ de especificidade.

Tabela 5 - Análise da sensibilidade e especificidade dos questionários ESE, Berlim e STOP-BANG em predizer o risco para AOS.n=35. Belém-PA,2019.

\begin{tabular}{lllllll}
\hline & ESE & & Q. BERLIM & \multicolumn{2}{l}{ Q. STOP-BANG } \\
\hline Sensibilidade & 68.97 & 49.17 a 84.72 & 96.55 & 82.24 a 99.91 & 93.1 & 77.23 a 99.15 \\
Especificidade & 33.33 & 43.27 a 77.72 & 0.00 & 0.0 a 45.93 & 16.67 & 0.42 a 64.12 \\
Valorespreditivospositivos & 83.33 & 62.62 a 95.26 & 82.35 & 65.47 a 93.24 & 84.38 & 67.21 a 94.72 \\
Valorespreditivosnegativos & 18.18 & 22.83 a 51.78 & 0.00 & 0.0 a 97.50 & 33.33 & 0.84 a 90.57 \\
Razão de verossimilhança & 1.034 & & \multicolumn{3}{c}{0,965} & \\
\hline
\end{tabular}

Fonte: Andrade JS, et al.,2019.

\section{DISCUSSÃO}

Embora a Apnéia Obstrutiva do Sono (AOS) seja reconhecida há mais de 40 anos, é significativamente subdiagnosticada e, consequentemente, não investigada. Nas populações ocidentais, cerca de 2 a $4 \%$ dos homens de meia idade e 1 a $2 \%$ das mulheres de meia idade têm AOS, mas na maioria desses pacientes, a AOS não é detectada e, portanto, não é tratada (PEPPARD PE, et al.,2013).

No presente estudo foi possível definir diagnóstico de AOS em $83 \%$ dos participantes. Com relação ao gênero, o masculino foi mais frequente $(55,1 \%)$, sendo mais evidente com aumento da gravidade, $70 \%$ dos 
pacientes com AOS grave eram do sexo masculino. Diversos estudam demonstram uma maior prevalência da AOS entre os homens, confirmando o sexo como um importante fator de risco para a doença (PEPPARD PE, et al., 2013; PEÑAFIEL FS, et al., 2018; ZANCANELLA E, et al.,2012; MARTINEI T, et al.,2017).

Com relação à idade, observou-se que a maioria dos pacientes com AOS eram da sexta década de vida, ratificando a idade maior que 50 anos como fator risco para AOS (ZANCANELLA E, et al.,2012). Não houve diferença significativa entre a média de idade dos pacientes entre os grupos da AOS.

Além da idade mais avançada (> 50) e do gênero masculino, os marcadores da obesidade, em especial o índice de massa corpórea (IMC) aumentado e a circunferência cervical aumentada, são os principais preditores da presença da AOS (USMANI ZA, et al., 2013; HEINZER R, et al., 2015; MANNARINO MR, et al.,2012). No presente estudo, o IMC teve média de 32.6 ( \pm 4,9 DP), sem diferença significativa entre os grupos da AOS.

Com relação à circunferência cervical, a média foi de $39.7 \mathrm{~cm}( \pm 4,9 \mathrm{DP})$, com maior média no grupo AOS grave $(41.2 \mathrm{~cm})$, sem diferença estatisticamente significativa. Amra B, et al. (2018), encontraram associação significativa entre IMC e circunferência do pescoço, com AOS moderada e grave, tendo média de 40.83 de circunferência cervical, e 32.40 de IMC.

Associar a impressão subjetiva, que inclui a história clínica com exame físico, com o resultado polissonográfico, permite aumento de certeza diagnóstica de AOS (ZANCANELLA E, et al.,2012).Na avaliação da história clínica dos pacientes, as queixas mais frequentes nos pacientes adultos com AOS comparados com não apneicos, são presença de ronco, sufocamento noturno, sonolência diurna e apnéia noturnas (MANNARINO MR, et al., 2012).

Resultados semelhantes aos encontrados neste estudo: ronco (100\%), sonolência diurna (91.4\%) e despertares noturnos $(88.6 \%)$ foram os mais frequentes. A hipertensão arterial foi a principal comorbidade encontrada no estudo. Ter hipertensão resistente ao tratamento clínico é fator de risco de AOS na população brasileira, com sensibilidade de $44 \%$ (31- 58\%), especificidade de $91 \%$, segundo estudos de (PEDROSA RP,et al.,2011; MUXFELDT ES, et al., 2014).

$\mathrm{Na}$ avaliação polissonográfica, a média de AlH foi de 35,5/h. Chama atenção a presença de dessaturação, chegando a $90 \%$ nos pacientes com AOS grave. Índices elevados também foram achados nos estudos de (MARTINEI T, et al.,2017). O índice de microdespertares (IMD) esteve elevado ( $>10$ eventos $/ \mathrm{h}$ ) em todos os grupos de AOS, com média de $38 / \mathrm{h}( \pm 12 \mathrm{DP})$. A literatura nos mostra que esses dados são preocupantes, pois, seus efeitos cumulativos associados à fragmentação do sono geram distúrbios na rotina diária e predispõem a desordens cardiovasculares (MANNARINO MR, et al.,2012).

A LSR tardia, fenômeno que altera a fisiologia do sono, foi encontrada em $48,6 \%$ dos pacientes do estudo e em $45 \%$ dos pacientes com AOS grave. De forma semelhante, Martinei T, et al. (2017) encontraram LSR tardia em $51.19 \%$ dos pacientes com AOS grave.

Estima-se que mais de $80 \%$ das pessoas com AOS moderada a grave permaneçam sem diagnóstico (PEPPARD PE, et al., 2013). Assim, é necessária uma ferramenta de triagem para estratificar os pacientes com base em seus sintomas clínicos e fatores de risco antropométricos. Alguns questionários fáceis de usar foram desenvolvidos como alternativas de baixo custo a PSG para detectar a AOS.

Nos últimos anos, a precisão dos questionários do sono tem sido uma área de crescente interesse de pesquisa e vários estudos foram publicados sobre o assunto. Nesta pesquisa, avaliamos ainda a sensibilidade e especificidade de três questionários autorreferidos comparados com a polissonografia: a Escala de Sonolência de Epworth (ESE), o Questionário de Berlim e Questionário STOP-BANG, que fazem parte do protocolo de atendimento do ambulatório de sono do Hospital Bettina Ferro de Souza.

Neste estudo $83 \%$ possuíam AOS, enquanto $97.1 \%$, 91.4\%, e $68.6 \%$ foram classificados como de alto risco pelos questionários de Berlim, STOP-BANG e ESE, respectivamente. A ESE apresentou sensibilidade de $68.97 \%$ e especificidade de $33.33 \%$. O questionário STOP-BANG apresentou alta sensibilidade (93.1\%) e baixa especificidade (16.6\%) para predizer o risco para AOS. 
O questionário de Berlim foi o que apresentou maior sensibilidade (96.5\%), porém $0 \%$ de especificidade. El-sayed IH (2012) encontrou resultados semelhantes em que dos 234 pacientes selecionados $87,1 \%$ possuíam AOS, destes $93.3 \%, 95.5 \%$ e $68.3 \%$ foram classificados como de alto risco pelos questionários de Berlim, STOP-BANG e ESS, respectivamente.

Os questionários STOP-BANG e Berlim tiveram a maior sensibilidade para prever AOS $(97.55 \%$ e $91.67 \%$, respectivamente), mas com uma especificidade muito baixa para pacientes com AOS $(26.32 \%$, e $25 \%$, respectivamente), a ESE teve especificidade para prever AOS em torno de $75 \%$ e sensibilidade $72.55 \%$.

Prasad KT, et al. (2017) em seu estudo realizado com 210 pacientes com queixa de roncos, o questionário STOP-BANG foi o que apresentou maior sensibilidade para detectar AOS tanto no grupo leve, moderada ou grave $(89 \%, 93.4 \%$ e 96.2 , respectivamente), porém com especificidade baixa $(43.5 \%, 39.2 \%$ e $32.1 \%$ ). No Questionário de Berlim a maior sensibilidade foi no grupo AOS grave $(89.4 \%)$, sendo a especificidade baixa para qualquer severidade de AOS. A ESE teve baixa sensibilidade e especificidade em todos os grupos. No presente estudo a maior sensibilidade foi o questionário Berlim (96.5\%), e a especificidade de todos os questionários também foi baixa.

Amra B, et al. (2018),em seu estudo realizado com 400 pacientes, as sensibilidades de Questionário de Berlim, STOP-BANG e ESE foram $86.42 \%, 81.46 \%$ e $59 \%$, respectivamente. As especificidades de do Questionário de Berlim, STOP - BANG e ESE foram 52.94\%, $82.35 \%$ e $76.47 \%$, respectivamente. Concordando com o presente estudo no qual a sensibilidade de ESS foi menor que Questionário de Berlim e STOP- BANG.

Nesta pesquisa a especificidade foi bem mais baixa, provavelmente pela diferença de população estudada e o tamanho da amostra. Pataka A, et al. (2014), utilizou ESE, Questionário de Berlim, STOPBANG para avaliar a população em geral, encontrando sensibilidade do Questionário STOP-BANG e Questionário de Berlim de $96.2 \%$ e $84.4 \%$, respectivamente e ambos os testes com baixa especificidade, de forma semelhante aos achados do presente estudo.

Em outros estudos feitos usando questionário de Berlim e STOP-BANG na população em geral, ambos tiveram um bom valor preditivo para a triagem da AOS moderada e grave, contrastando comos resultados encontrados nesta pesquisa (TANYJD, et al., 2016; TAN A, et al., 2017). Uma diferença importante é que neste estudo não foi avaliado a população em geral e sim em pacientes que foram encaminhados ao ambulatório do sono devido a queixas de ronco.

Pacientes sem diagnóstico de AOS foram poucos. Esse status pré-selecionado da população provavelmente afetou os resultados. O fato de que a AOS é altamente prevalente em pacientes com distúrbios do sono, pode resultar em aumento acentuado na sensibilidade aparente dos questionários e redução da especificidade.

Tanto nos resultados desta pesquisa quanto na literatura, a exemplo dos estudos já elencados, verificase que a ESE apresenta especificidade e sensibilidade baixas para predizer o risco de AOS. Isto não é surpreendente porque a ESE é um questionário padrão para medir subjetivamente a sonolência diurna excessiva, que é um critério de diagnóstico para AOS, mas que pode ocorrer secundário a várias causas diferente de AOS e a doenças que provoquem fragmentação e alterações no tempo de sono (JOHNS MW, 1991).

\section{CONCLUSÃO}

Neste estudo a prevalência de AOS foi de $83 \%$, sendo a maioria classificada com AOS grave. Idade mais avançada, gênero masculino, índice de massa corpórea aumentado e a circunferência cervical aumentada, foram encontrados no estudo, ratificando os principais preditores da AOS. Na AOS grave, o gênero masculino foi predominante, não havendo diferença estaticamente significativa quanto a IMC, idade e perímetro cervical quanto a gravidade da AOS.Os questionários utilizados: ESE, Berlim e STOP-BANG, foram capazes de identificar pacientes de alto risco para AOS, mas sem excluir com precisão aqueles com baixo risco. 


\section{REFERÊNCIAS}

1. AMRA B, et al. Screening Questionnaires for Obstructive Sleep Apnea: An Updated Systematic Review. Oman Medical Journal, 2018; 3: 184-192.

2. AMRA B, et al. Comparison of obstructive sleep apnea questionnaires. International Journal of Preventive Medicine, 2018; 9: 28

3. BERRY RB, et al. for the American Academy of Sleep Medicine. The AASM Manual for the Scoring of Sleep and Associated Events: Rules, Terminology and Technical Specifications, Version 2.5. American Academy of Sleep Medicine, 2018.

4. BIXLER EO, et al. Prevalence of sleep-disordered breathing in women: effects of gender. Am J Respir Crit Care Med. 2001;163(3):608-13.

5. DUARTE RLM, et al. Validação do questionário STOP-Bang para a identificação de apnéia obstrutiva do sono em adultos no Brasil. J Bras Pneumol. 2017;43(6):456-463

6. EL-SAYED IH. Comparison of four sleep questionnaires for screening obstructive sleep apnea. Egyptian Journal of Chest Diseases and Tuberculosis, 2012; (61): 433-441

7. HEINZER R, et al. Prevalence of sleep-disordered breathing in the general population: the Hypno Laus study. Lancet Respir Med, 2015;3(4):310- 8

8. IP MS, et al. A community study of sleep-disordered breathing in middle-aged Chinese women in Hong Kong: prevalence and gender differences. Chest. 2004;125(1):127-34.

9. JOHNS MW. A new method for measuring daytime sleepiness: the Epworth sleepiness scale. Sleep, 1991; (14): 540-545.

10. KIM J, et al. Prevalence of sleep-disordered breathing in middle-aged Korean men and women. Am J Respir Crit Care Med. 2004;170(10):1108-13.

11. MANNARINO MR, et al. Obstructive sleep apnea syndrome. Eur J InternMed, 2012; 23: 586-93.

12. MARTINEI T, et.al. Correlação da ESE com a polissonografia na avaliação de SED. Medicina(Ribeirão Preto, Online.), 2017;50(2):102-8

13. MUXFELDT ES, et al. Prevalence and associated factors of obstructive sleep apnea in patients with resistant hypertension. Am J Hypertens. 2014;27(8):1069-78.

14. NAGAPPA M, et al. Validation of the STOP- Bang questionnaire as a screening tool for obstructive sleep apnea among different populations: A systematic review and meta-analysis. PLoSOne. 2015; 10: e014369

15. PARK JG, et al. Updates on definition,consequences, and management of obstructive sleepapnea. Mayo Clin Proc. 2011; (86): 549-54.

16. PATAKA A, et al. Evaluation of Five different questionnaires for assessing sleep apnea syndrome in a sleep clinic. $J$ Clin Sleep Med, 2014; (15):77681.

17. PEDROSA RP, et al. Obstructive sleep apnea: the most common secondary cause of hypertension associated with resistant hypertension. Hypertension, 2011; (58):811-7.

18. PEÑAFIEL FS, et al. Evaluación de los cuestionarios de sueño em la pesquisa de pacientes con síndrome de apneas obstructivas del sueño. RevMed Chile, 2018; 146: 1123-1134

19. PEPPARD PE, et al. Increased prevalence of sleep-disordered breathing in adults. Am J Epidemiol,2013; (177): $1006-14$.

20. PRASAD KT, et al. Assessing the likelihood of obstructive sleep apnea: a comparison of nine screening questionnaires. Sleep Breath, 2017; (4):909-917.

21. TAN A,et al. Using the Berlin questionnaire to predict obstructive sleep apnea in the general population. J Clin Sleep Med, 2017;(13):42732.

22. TAN YJD, et al. Predicting obstructive sleep apnea using the STOP-Bang questionnaire in the general population. J Clin Sleep Med, 2016;2728:6671.

23. TUFIK S, et al. Obstructive sleep apnea syndrome in the Sao Paulo Epidemiologic Sleep Study. Sleep Med. 2010;11(5):441-6.

24. USMANI ZA, et al. Obstructive sleep apnea in adults. Postgrad MedJ, 2013;89:148-156.

25. ZANCANELLA E, et al. Apnéia Obstrutiva do Sono e Ronco Primário: Diagnóstico. Projeto diretrizes- Associação Médica Brasileira e Conselho Federal de Medicina, 2012. 